\title{
Application of FRET microscopy for visualizing the uncoating process of a single Dengue virus in living cells
}

\author{
Li-Wei Chu ${ }^{1,3,4}$, Ya-Hsuan Lin ${ }^{1,3,4}$, Chau-Hwang Lee ${ }^{1,3,5}$, and Yueh-Hsin Ping ${ }^{1,2,3,4}$ \\ 1. Institute of Biophotonics, National Yang-Ming University, Taipei, Taiwan. \\ 2. Department and Institute of Pharmacology, National Yang-Ming University, Taipei, Taiwan. \\ 3. Biophotonics interdisciplinary Research Center, National Yang-Ming University, Taipei, Taiwan. \\ 4. Infection and Immunity Research Center, National Yang-Ming University, Taipei, Taiwan. \\ 5. Research Center for Applied Sciences, Academia Sinica, Taipei, Taiwan.
}

Single-virus tracking is a real-time imaging technique that can monitor successfully individual virus entry and trafficking behavior in live cells [1]. Conventional wide-field fluorescence microscopy for singlevirus tracking enables localization of fluorescently labeled molecules within the optical spatial resolution limits defined by the Rayleigh criterion, approximately 200 nanometers. However, the scale of virus uncoating involved virus-host membrane fusion is within dozens nanometer. It is smaller than optical resolution limitation and failed to detect by single-particle tracking. In order to resolve this limitation, the Förster resonance energy transfer (FRET), when applied to optical microscopy, permits determination of the approach between two molecules within several nanometers [2]. It can be useful to determine the virus-host membrane fusion in viral uncoating events. In our previous study, using a single-virus particle tracking technology demonstrated that dengue virus (DENV) particles interacted directly with autophagosomes, and autophagy facilitated viral particle trafficking and promoted viral replication [3]. Herein, we established the FRET pair fluorophore labeling DENV particles using DiI as a FRET donor and DiD as a FRET acceptor. We hypothesized that the distances of DiI and DiD molecules in virus envelope are elevated during viral uncoating process, resulting in the decreasing of FRET efficiency (Figure 1A). The decreasing FRET signals can be considered as viral uncoating. To characterize the properties of DiI/DiD-labeled DENV, the fluorescent intensity and the FRET effect of DiI/DiD-labeled virus were measured by confocal microscopy and acceptor-photobleaching imaging (Figure1B\&C). The FRET efficiency of DiI/DiD-labeled virus particles is about 0.75 (Figure 1C). After 30 min postinfection for the internalization of DENV, the DENV particles that colocalized with autophagosomes (green puncta) presented lower FRET efficiency in FRET accptor-bleaching imaging (Figure 2, white arrow), indicating that DENV uncoating occured in autophagosomes. In addition, we also measured the alteration of fluorescence intensity of individual DENV particles in living cells by single-particle tracking. In this expirement, the $532 \mathrm{~nm}$ laser was used to excite the FRET donor, DiI, and both DiI and DiD signal were recorded to caculate the emision ratio of DiD/DiI. The $488 \mathrm{~nm}$ laser was used to excite GFP-LC3 fusion protein, as a marker of autophagosome. The results showed that DENV particles co-transported with autophagosomes (green puncta) and presented the decreasing ratio of DiD/DiI suggesting that DENV uncoating is correlated the colocalization of viral particle with autophagosome (Figure 3). In conclusion, we utilized lipophilic DiI and DiD dye to generate FRET pair fluophores labeling DENV particles. This approach offers an opportunity to reveal DENV uncoating process in living cells at singlevirus level by FRET microscopy. Our results elucidated that autophagy facilitates the uncoating process of DENV. Furthermore, the combination of FRET and a single-virus tracking approaches not only provides more detail of virus-host interaction, but also can be further develop as a screening platform for developing anti-viral drugs.

[1] B. Brandenburg, X. Zhuang, Virus trafficking - learning from single-virus tracking, Nat Rev 
Microbiol 5 (2007) 197-208.

[2] J. Zheng, Spectroscopy-based quantitative fluorescence resonance energy transfer analysis, Methods Mol Biol 337 (2006) 65-77.

[3] L.W. Chu et al, Single-virus tracking approach to reveal the interaction of Dengue virus with autophagy during the early stage of infection, J Biomed Opt 19 (2014) 011018.
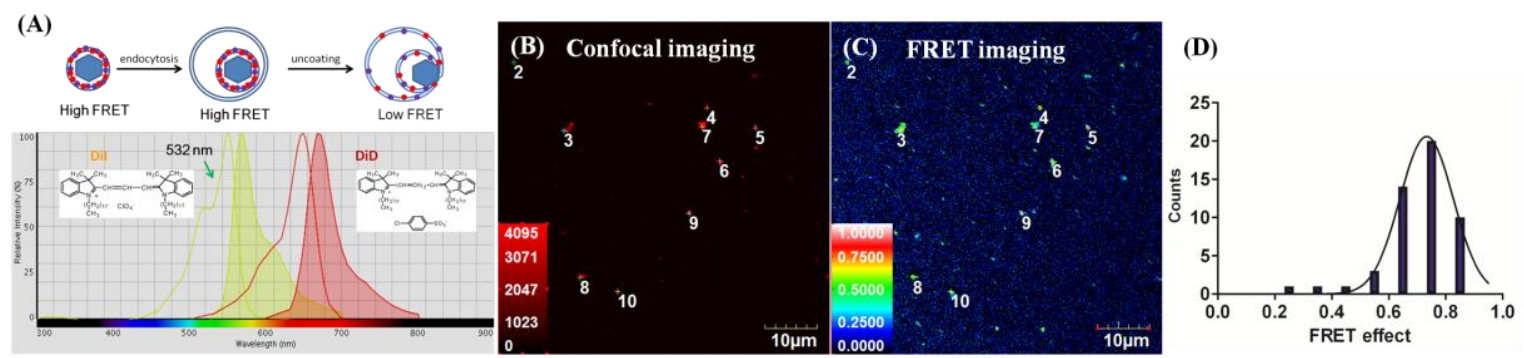

Figure 1. (A) Scheme of DiI \& DiD labeling as FRET pair for visualizing DENV uncoating. Characterization of DiI-DiD labeled DENV represented by (B) Confocal and (C) FRET Acceptor photoleaching imaging. (D) Quantification of FRET efficiency of DENV particles ( $\mathrm{n}=50)$.

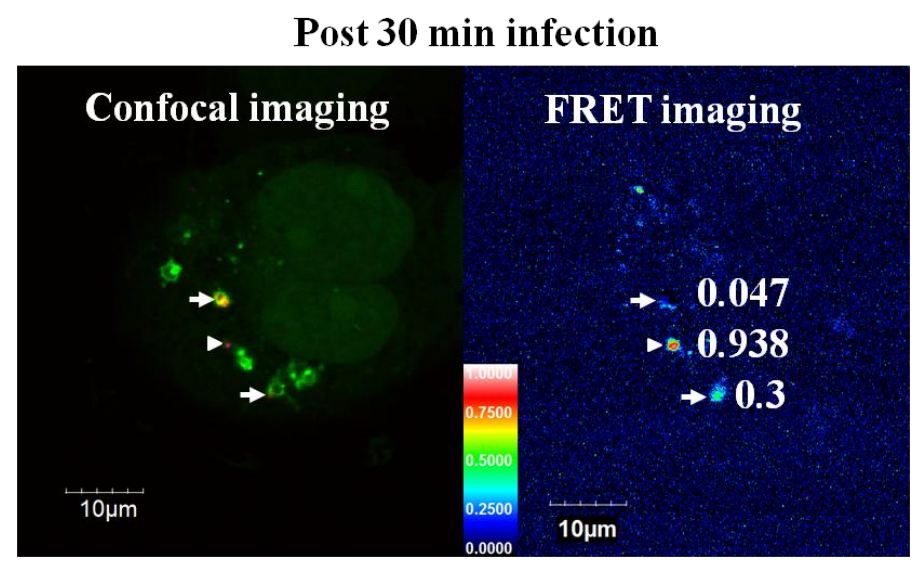

Figure 2. DENV colocalized with autophagosomes and occurred to FRET signal decay (white arrow). When DENV didn't colocalize with autophagosomes, it kept high FRET signal (arrow head).
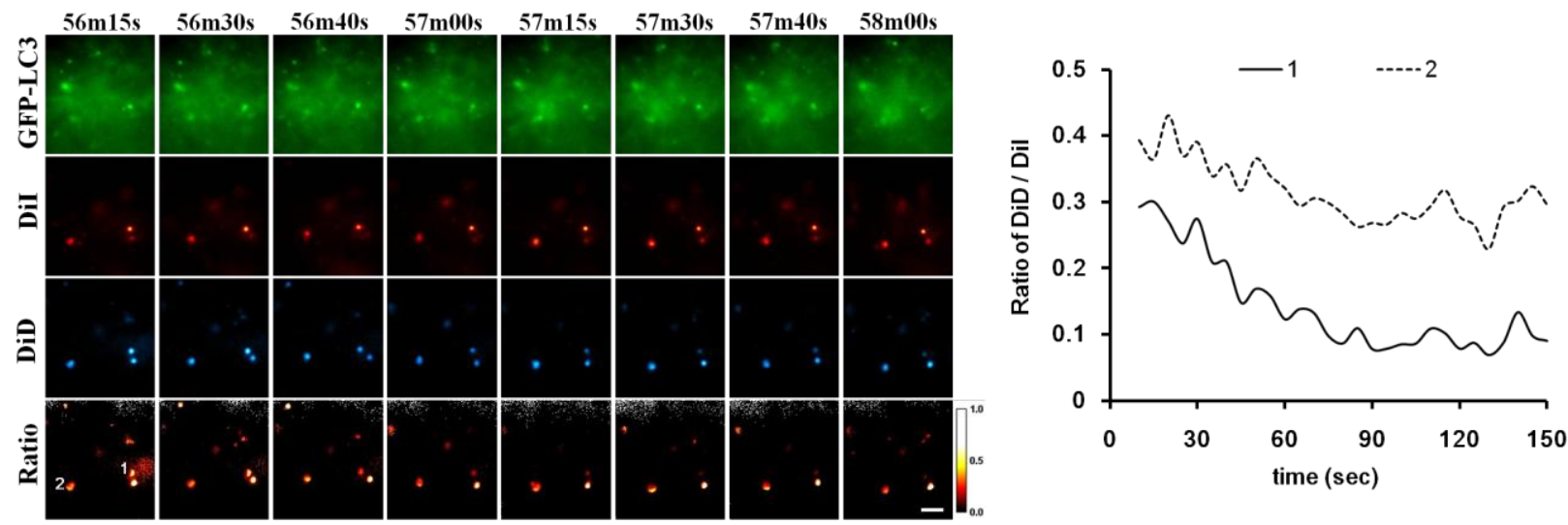

Figure 3. FRET microscopy applied to single-virus tracking for visualizing individual DENV uncoating event in living cells. Scale bar: $2 \mu \mathrm{m}$. 